\title{
Respuesta de semillas de tres especies nativas altoandinas a diferentes condiciones de almacenamiento
}

\section{Seeds response to different storage conditions of three highland native tree species}

\author{
Claudia Patiño-Uyaguari ${ }^{1}$, Janeth Jiménez-Sánchez ${ }^{1}$, Franklin Marín-Molina ${ }^{2}$ iD , Ximena \\ Palomeque-Pesántez $z^{1,2}$ (i) \\ ${ }^{1}$ Facultad de Ciencias Agropecuarias, Carrera de Ingeniería Agronómica, Universidad de Cuenca, Cuenca, Ecuador. \\ 2 Departamento de Recursos Hídricos y Ciencias Ambientales, Universidad de Cuenca, Cuenca, Ecuador. \\ Autor para correspondencia: ximena.palomeque@ucuenca.edu.ec \\ Fecha de recepción: 3 de octubre de 2019 - Fecha de aceptación: 10 de diciembre de 2019
}

\section{RESUMEN}

Para asegurar la capacidad de germinación y viabilidad de especies forestales nativas es importante conocer las condiciones óptimas para el almacenamiento de semillas a largo plazo. En respuesta a esto, nosotros investigamos el efecto del almacenamiento de semillas en la germinación, viabilidad y velocidad de germinación (VG) de Vallea stipularis, Hedyosmum luteynii y Oreopanax avicenniifolius. Las semillas fueron colectadas de los bosques Llaviucu y Mazán del Parque Nacional El Cajas y fueron expuestas a diferentes tratamientos de almacenamiento: tres contenidos de humedad (inicial, medio bajo), dos temperaturas $\left(10^{\circ} \mathrm{C}\right.$ y temperatura ambiente) y tres tiempos de almacenamiento (3, 6 y 12 meses). Los resultados mostraron que en general las semillas de $V$. stipularis almacenadas a $10^{\circ} \mathrm{C}$ durante 12 meses, presentaron bajos e incluso nulos porcentajes de geminación; la viabilidad y VG disminuía conforme se incrementaba el tiempo de almacenamiento. Las semillas de $H$. luteynii tuvieron una alta germinación y viabilidad hasta los 12 meses de almacenamiento a $10^{\circ} \mathrm{C}$, sin embargo, su germinación fue tardía y poco sincronizada. El almacenamiento a temperatura ambiente no favoreció la germinación y VG de las dos especies, aunque, $H$. luteynii mantuvo su viabilidad intacta hasta el último periodo evaluado. $O$. avicenniifolius mantuvo su capacidad germinativa hasta los 12 meses de almacenamiento, tanto a $10^{\circ} \mathrm{C}$ como temperatura ambiente, pero con baja germinación. El conocimiento generado contribuye para un mejor manejo de las semillas, en programas de conservación $e x$ situ y la producción de plántulas para la restauración.

Palabras claves: Tiempo de almacenamiento, contenido de humedad, temperatura, Parque Nacional El Cajas, Andes.

\begin{abstract}
To ensure the germination capability and viability of native tree species it is important to know the optimum conditions for seed storage in the long term. In response to this, we investigated the effect of seed storage on the germination, viability, and velocity of germination (VG) of Vallea stipularis, Hedyosmum luteynii and Oreopanax avicenniifolius. The seeds were collected in Llaviucu and Mazan forests in Cajas National Park and exposed to different treatments of seed storage: three levels of seed moisture content (initial, medium and low), two levels of temperature $\left(10^{\circ} \mathrm{C}\right.$ and room temperature) and three periods of storage (3, 6, and 12 months). The research revealed that seeds of $V$. stipularis stored at $10^{\circ} \mathrm{C}$ for 12 months had the lowest germination values, even null percentages; the more the seed viability and VG decreased when the time of seed storage increased. The seeds of $H$. luteynii showed high germination and viability up to 12 months of storage at $10^{\circ} \mathrm{C}$, however, its germination was later and little synchronized. The room temperature did not work well for germination and VG for both these species, although, $H$. luteynii maintained its viability intact up to the last period of evaluation. O. avicenniifolius maintained at $10^{\circ} \mathrm{C}$ and room temperature its germination capacity up to 12 months but with a lower percentage. The knowledge generated contributes to better seed management in ex situ conservation programs and plant propagation for restoration.
\end{abstract}

Keywords: Time of storage, seed moisture content, temperature, Cajas National Park, Andes.

\section{INTRODUCCIÓN}

Ecuador es considerado uno de los países megadiversos, por la presencia de una gran variedad de formas de vida en las cuatro regiones del país (Grijalva et al., 2015), siendo la región andina considerada como uno de los puntos calientes de biodiversidad en el mundo (Myers, Mittermeier, Mittermeier, da Fonseca, \& Kent, 2000). El $34.7 \%(9,599,678$ ha) de la superficie de este país está constituida por ecosistemas forestales (FAO, 2012). Sin embargo, esta área de bosque se está reduciendo por el cambio de uso de suelo, por la deforestación de vegetación 
nativa para el establecimiento de tierras de uso agrícola (Estrella, Manosalvas, Mariaca, \& Ribadeneira, 2005), y debido a la ampliación de las ciudades por el crecimiento poblacional (Defries, Rudel, Uriarte, \& Hansen, 2010).

Hoy en día, hay una creciente preocupación por la pérdida y degradación de los bosques y su biodiversidad asociada, de ahí que, el Ecuador se ha comprometido en restaurar paisajes forestales dentro de la iniciativa 20x20, con una contribución de 500,000 hectáreas dentro de la Convención Marco de las Naciones Unidas en el 2014. Además, se cuenta con un Plan Nacional de Restauración Forestal para el 2019-2030 (Ministerio del Ambiente, 2019). Para cumplir con los propósitos enmarcados a nivel internacional y nacional, se requiere de técnicas apropiadas que en muchos de los casos dependen de la calidad de las semillas, como por ejemplo la propagación de plantas de calidad para la reforestación (Smith, Mengistu, Nelson, \& Paris, 2008; Palomeque et al., 2017). Sin embargo, entre los principales inconvenientes en las semillas, es la limitada información de la gran variedad de especies potenciales para la restauración, que incluye desde la identificación de fuentes semilleras, calidad de las semillas, consideraciones genéticas, dormancia, tratamientos pregerminativos, germinación y su velocidad, establecimiento de plántulas y almacenamiento de semillas (Kindt et al., 2006; Palomeque et al., 2017). Un aspecto crucial en la ejecución de los programas de reforestación, es mantener la cantidad y viabilidad de semillas en el mayor tiempo posible (Doria, 2010), considerando que las épocas de fructificación de los árboles, no necesariamente coinciden con los cronogramas de producción de plantas por parte de los viveristas locales (Velasquez, Montero, \& Tapia, 2008) o a través de instituciones gubernamentales y no gubernamentales; de ahí la importancia de estudiar las condiciones óptimas de almacenamiento para cada especie.

Varios autores afirman que el almacenamiento de las semillas depende si son de tipo ortodoxas, recalcitrantes o intermedias (Pammenter \& Berjak, 2000; Schmidt, 2000). Las semillas ortodoxas poseen contenido de humedad relativamente bajo $(<15 \%)$ y toleran una deshidratación hasta de 5\% (Gentil, 2001) y pueden ser almacenadas hasta temperaturas bajo cero, durante muchos años hasta décadas o siglos, dependiendo de la especie (Walters, Wheeler, \& Grotenhuis, 2005). Las semillas recalcitrantes tienen alto contenido de humedad (sensibles a la desecación), pierden viabilidad cuando su contenido de humedad cae por debajo del 20-30\% (Pritchard, 2004), y no pueden ser almacenadas a temperaturas extremadamente bajas y durante tiempos prolongados (Schmidt, 2000); mientras que semillas intermedias presentan características de ortodoxas y recalcitrantes (Royal Botanic Gardens Kew, 2015). A partir de esta información se puede establecer de antemano las condiciones necesarias de almacenamiento tales como: temperatura, contenidos de humedad de semilla y tiempo de almacenamiento. Todos estos factores al integrarlos hacen que el entendimiento de la respuesta de las semillas en términos de germinación, viabilidad y velocidad de germinación sea muy complejo.

Estudios demuestran que, al integrar la temperatura y contenido de humedad de la semilla, modulan la tasa de envejecimiento de la semilla y en consecuencia reduce el potencial germinativo y su viabilidad (Pritchard \& Dickie, 2003; Walters et al., 2005; Bewley, Bradford, Hilhorst, \&
Nonogaki, 2013). Otro ejemplo que ilustra la complejidad es el almacenamiento de semillas a largo plazo, incluso cuando éstas se almacenan a temperaturas con niveles de humedad y oxígeno óptimos (Pukacka \& Ratajczak, 2007); normalmente la capacidad de germinación y el vigor se pierde durante el curso de envejecimiento, y consecuentemente hay una pérdida de plántulas (Çakmak, Atici, Agar, \& Sunar, 2010).

Otro enfoque del estudio sobre almacenamiento de semillas de especies nativas es la contribución a la conservación ex situ, en bancos de germoplasma (BG), los mismos que tienen a su cargo la conservación, producción y el uso del conocimiento sobre semillas comestibles, forestales, medicinales, ornamentales en el mundo (FAO, FIDA, \& PMA, 2014). En Ecuador, el BG público manejado por el Instituto Nacional Autónomo de Investigaciones Agropecuarias (INIAP), cuenta con 88 accesiones de semillas de especies forestales, que incluyen especies exóticas (Romero-Saritama, 2018).

Este estudio se realizó con el fin de responder a la pregunta ¿Cuál es la capacidad germinativa, viabilidad y velocidad de germinación de semillas almacenadas en diferentes condiciones de almacenamiento (temperatura, contenido de humedad de la semillas y tiempo de almacenamiento) para las especies forestales nativas Vallea stipularis, Hedyosmum luteynii y Oreopanax avicenniifolius? La información generada aporta con protocolos de almacenamiento de semillas forestales de especies altoandinas para su conservación y uso en programas de restauración a través de la reforestación o siembra directa de semillas con especies nativas.

\section{MATERIALES Y MÉTODOS}

\section{1. Área de estudio y colección de semillas}

Se utilizó semillas de tres especies forestales nativas: Vallea stipularis L. f. (Sacha capulí), Hedyosmum luteynii Todzia (Borracho) y Oreopanax avicenniifolius (Kunth) Decnce. \& Planch. (Pumamaqui), provenientes de los bosques de Llaviucu y Mazán del Parque Nacional El Cajas, provincia del Azuay (Fig. 1).

Ambos bosques están ubicados a una altitud desde 3103 hasta 3178 m.s.n.m. y corresponden al ecosistema bosque Siempre Verde Montano Alto (Sierra, 1999). Las semillas de cada especie, colectadas de frutos maduros de al menos 10 árboles por bosque, con las mejores características fenotípicas. La colección de los frutos se realizó dependiendo del periodo de fructificación de cada especie: V. stipularis entre mayo y junio del 2017 , H. luteynii entre enero y febrero del 2018 y O. avicenniifolius entre febrero y abril del 2018. Para cada especie, las semillas de los dos bosques fueron mezcladas con el fin de garantizar la variabilidad genética.

\subsection{Reducción del contenido de humedad de las semillas y tratamientos de almacenamiento}

Los ensayos se realizaron en el laboratorio de Ecología Forestal y Semillas, de la Universidad de Cuenca. El cálculo del contenido de humedad inicial de las semillas por cada especie se basó en las normas de la Asociación Internacional de Ensayos de Semillas (ISTA, 2007), para lo cual se utilizó dos repeticiones de 100 semillas cada 

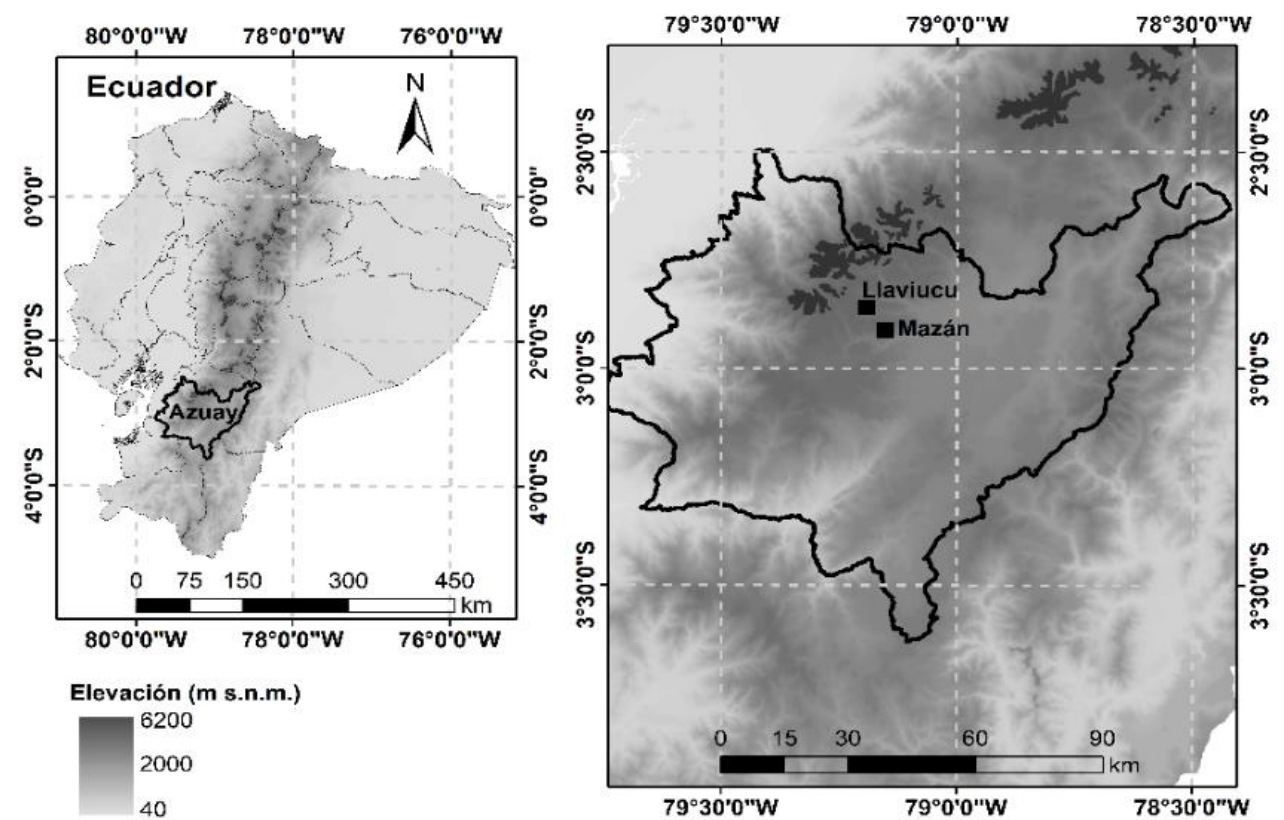

Figura 1. Fuentes semilleras de los bosques de Mazán y Llaviucu del Parque Nacional El Cajas en la provincia del Azuay, Ecuador.

Tabla 1. Intervalos de tiempo para lograr los diferentes rangos de los contenidos de humedad de semillas para el almacenamiento de tres especies forestales nativas.

\begin{tabular}{lcc}
\hline Especies & Intervalo de tiempo de desecación & Contenido de humedad (\%) \\
\hline \multirow{2}{*}{ Vallea stipularis } & $1020 \mathrm{~min}$ & Inicial (10.17) \\
& $23 \mathrm{~min}$ & Medio (8) \\
\hline \multirow{2}{*}{ Hedyosmum } & $173 \mathrm{~min}$ & Bajo (6) \\
luteynii & $1020 \mathrm{~min}$ & Inicial (10.38) \\
\hline \multirow{2}{*}{ Oreopanax } & $16 \mathrm{~min}$ & Medio (8) \\
avicenniifolius & $40 \mathrm{~min}$ & Bajo (5) \\
\hline Fuente: Jiménez \& Patiño $(2019)$ & $1020 \mathrm{~min}$ & Inicial (9.47) \\
\end{tabular}

Tabla 2. Métodos de desinfección empleados para el control sanitario en las semillas de las diferentes especies.

\begin{tabular}{ll}
\hline Especies & \multicolumn{1}{c}{ Método de desinfección } \\
\hline Vallea stipularis & $\begin{array}{l}\text { Fungicida Clorhexidina + Cetrimide (Germinal) al 10\% durante 2 minutos } \\
\text { y enjuague con agua destilada. }\end{array}$ \\
\hline $\begin{array}{l}\text { Hedyosmum } \\
\text { luteynii }\end{array}$ & Agua Destilada + Jabón líquido y enjuague con agua destilada. \\
\hline $\begin{array}{l}\text { Oreopanax } \\
\text { avicenniifolius }\end{array}$ & $\begin{array}{l}\text { Hipoclorito de sodio al 5,25\% (Clorox) durante 2 minutos y enjuague con } \\
\text { agua destilada. }\end{array}$ \\
\hline Fuente: Jiménez \& Patiño (2019)
\end{tabular}

una, considerando el peso antes y después de secar en un horno a $103^{\circ} \mathrm{C}$ durante 17 horas. A partir del contenido de humedad inicial de las tres especies, un lote de semillas con dos repeticiones de 100 semillas fue utilizado para generar una curva de desecación, para definir dos niveles de contenido de humedad (medio y bajo), para lo cual se utilizó una estufa con temperatura constante a $45^{\circ} \mathrm{C}$ y por diferentes periodos de tiempo hasta determinar el tiempo específico y el contenido de humedad deseado (Tabla 1); este fue un proceso destructivo para las semillas. Para los respectivos cálculos se utilizó la siguiente ecuación (ISTA, 2007):

$$
C H=(m 2-m 3) * \frac{100}{(m 2-m 1)}
$$

donde $\mathrm{CH}$ es el contenido de humedad (\%) de la semilla; $m 1$ el peso en gramos del contenedor y su cubierta; $m 2$ el peso en gramos del contenedor, su cubierta y las semillas antes del secado, y $\mathrm{m} 3$ el peso en gramos del contenedor, su cubierta y las semillas después del secado.

Para el experimento del almacenamiento de semillas se utilizaron tres factores: 1) contenido de humedad (inicial, medio y bajo), 2) temperatura (temperatura ambiente y $\left.10^{\circ} \mathrm{C}\right)$, y 3$)$ tiempo de almacenamiento (3, 6 y 12 meses). 
Una vez alcanzados los niveles de contenido de humedad deseados de las semillas para cada especie, estas fueron almacenadas en papel aluminio y fundas ziploc con silica gel (absorbente de humedad) y colocados en recipientes de vidrio totalmente herméticos, para evitar que las semillas absorban humedad. Para evaluar la influencia de la temperatura sobre el almacenamiento, se utilizó una refrigeradora calibrada a $10^{\circ} \mathrm{C}$ y para el almacenamiento a temperatura ambiente (promedio $21^{\circ} \mathrm{C}$ ), se utilizó un estante de vidrio hermético ubicado en el laboratorio. Se aplicó un diseño completamente al azar, y por cada tratamiento se utilizó cuatro repeticiones con 25 semillas cada una. En total se tuvo 72 unidades experimentales por especie. Adicionalmente, se evaluó la germinación de las semillas sin almacenamiento aplicando solamente los tres niveles de humedad antes descritos.

\subsection{Pruebas de germinación, viabilidad y cálculo de la velocidad de germinación}

Una vez trascurridos los diferentes periodos de almacenamiento de cada especie, se evaluó la germinación en porcentaje, colocando las semillas en cajas Petri de vidrio con papel toalla previamente esterilizados. Además, en línea con previos ensayos se utilizó un método de desinfección específico para las semillas de cada especie, el mismo que se especifica en la Tabla 2. Para mantener la humedad se utilizó agua destilada durante todo el proceso de germinación y su monitoreo se realizó cada dos días durante 75 días para $V$. stipularis, 55 días para $H$. luteynii y 35 días para $O$. avicenniifolius, contando desde el día en que inicia la geminación. Se consideró como semilla germinada cuando la nueva plántula presentaba sus primeras hojas cotiledóneas (ISTA, 2007). Al final de la etapa de germinación de los diferentes tratamientos aplicados, las semillas que no germinaron fueron evaluadas su viabilidad utilizando sales de Tetrazolio al 1\%, para observar la tinción del embrión (ISTA, 2007). Además, se categorizó a las semillas como podridas y vacías, para lo cual la semilla fue cortada. Estos resultados fueron expresados en porcentaje.

La velocidad de germinación fue calculada a partir de los registros de germinación. El coeficiente de velocidad (CVG) se calculó a partir de la siguiente ecuación (Baskin \& Baskin, 2014):

$$
C V G=\frac{100(A 1+A 2+\cdots+A x)}{(A 1 . T 1+A 2 . T 2+\cdots+A x . T x)}
$$

donde $C V G$ es el coeficiente de velocidad germinativa; $A 1, A 2, A x$ son los números de semillas contadas desde el primer día, segundo día hasta el último día, etc.; y $T 1, T 2$, $T x$ el número de días entre la siembra y el primer día de registro de germinación, segundo día de registro de germinación hasta el último día de registro.

\subsection{Análisis estadístico}

El efecto de las diferentes condiciones de almacenamiento de cada especie, sobre la geminación, viabilidad, pudrición, semillas vacías y coeficientes de velocidad de germinación, se evaluó utilizando los modelos lineales generalizados (GLM). Se consideró como factores fijos al tiempo, contenido de humedad y temperatura de

${ }^{1}$ nlme: Linear and Nonlinear Mixed Effects Models almacenamiento con sus combinaciones. Para la selección de los mejores modelos se utilizó su bondad de ajuste del modelo a través del criterio de información de Akaike (AIC) y del criterio de información Bayesiano (BIC). Adicionalmente, se evaluó la normalidad de sus residuales mediante la prueba de Kolmogorov-Smirnov. Los modelos que no presentaron una distribución normal fueron transformados mediante la raíz cuadrada. Los GLM, fueron analizados por especie, utilizando las librerías nlme ${ }^{1}$ (Pinheiro, Bates, DebRoy, \& Sarkar,2018) y $\operatorname{lme}^{2}$ (Bates, Machler, Bolker, \& Walker, 2015) del programa estadístico R 3.5.1 (R Core Team, 2018). Un análisis de varianza (ANDEVA) fue realizado para determinar las diferencias estadísticas de las semillas con sus distintos niveles de $\mathrm{CH}$, que no fueron sometidas almacenamiento y se aplicó la prueba Tukey $(\mathrm{p}<0.05)$ para encontrar diferencias entre las medias de los grupos.

\section{RESULTADOS}

\subsection{Germinación y viabilidad inicial de semillas sin almacenamiento}

El porcentaje de germinación inicial de $V$. stipularis, en los tres niveles de contenido de humedad $(\mathrm{CH})$ evaluados antes del almacenamiento, osciló entre 48 y $57 \%$, y su viabilidad fue inferior al 25\%. Para $H$. luteynii, el porcentaje de germinación varió entre 37 y $50 \%$ y la viabilidad entre $26 \%$ y $37 \%$. La germinación inicial de $O$. avicenniifolius fue menor al 35\%, así como la viabilidad estuvo por debajo del 5\% (Tabla 3). En general, las tres especies presentaron bajo porcentajes de semillas vacías y para la variable pudrición; $O$. avicenniifolius tuvo porcentajes superiores al $61 \%$ mientras que las dos especies reportaron valores inferiores al $17 \%$. En cuanto a la velocidad de germinación, la especie que presentó una germinación más temprana, rápida y sincronizada fue $O$. avicenniifolius con un CVG máximo de 2.3, y contrariamente $H$. luteynii tuvo una germinación tardía, lenta y desincronizada con valores máximos de CVG de 0.9 . V. stipularis mostró un comportamiento de velocidad de germinación intermedio con un CVG máximo de 1.4, con respecto a las dos especies. No se encontró diferencias estadísticamente significativas entre los diferentes niveles de $\mathrm{CH}$ de las semillas no almacenadas para las tres especies en las variables analizadas, excepto para $O$. avicenniifolius en la variable semillas vacías (Tabla 3 ).

\subsection{Efecto de las diferentes condiciones de almacenamiento sobre el porcentaje de germinación de tres especies forestales nativas}

Las semillas de $V$. stipularis almacenadas a $10^{\circ} \mathrm{C}$ con los tres niveles de $\mathrm{CH}$, tanto a los 3 como 6 meses, presentaron similares porcentajes de germinación, entre 50 y $65 \%$ (Fig. 2a,b) y a los 12 meses, la germinación se redujo drásticamente $(<6 \%)$ e incluso nula en el tratamiento de almacenamiento con $6 \% \mathrm{CH}$ (Fig. 2c). Las semillas almacenadas a temperatura ambiente, a partir del tercer mes presentaron una disminución severa de la germinación, inferior al 25\% (Fig. 2a); para el sexto mes solamente el tratamiento de almacenamiento con $6 \%$ de

\footnotetext{
${ }^{2}$ lme4: Mixed-effects models in $\mathrm{R}$
} 
Tabla 3. Media de los porcentajes iniciales de germinación, viabilidad, semillas podridas y vacías, y promedio del coeficiente de velocidad de germinación (CVG) de semillas sin almacenamiento de tres especies forestales con tres niveles de contenidos de humedad de las semillas.

\begin{tabular}{|c|c|c|c|c|c|c|c|c|c|c|c|}
\hline \multirow[t]{2}{*}{ Especie } & \multirow{2}{*}{$\begin{array}{l}\mathrm{CH} \\
(\%)\end{array}$} & \multicolumn{2}{|c|}{$\begin{array}{c}\text { Germinación } \\
(\%)\end{array}$} & \multicolumn{2}{|c|}{ Viabilidad (\%) } & \multicolumn{2}{|c|}{ Vacías (\%) } & \multicolumn{2}{|c|}{ Podridas (\%) } & \multicolumn{2}{|c|}{$\mathrm{CVG}$} \\
\hline & & media & $\mathrm{DE}$ & media & $\mathrm{DE}$ & media & $\mathrm{DE}$ & media & $\mathrm{DE}$ & media & $\mathrm{DE}$ \\
\hline \multirow{3}{*}{$\begin{array}{c}\text { Vallea } \\
\text { stipularis }\end{array}$} & 6 & $48 \mathrm{a}$ & 12.65 & $25 \mathrm{a}$ & 3.83 & $3 a$ & 6.00 & $17 \mathrm{a}$ & 6.00 & $1.40 \mathrm{a}$ & 0.11 \\
\hline & 8 & $49 \mathrm{a}$ & 8.87 & $21 \mathrm{a}$ & 3.83 & $3 a$ & 3.83 & $16 \mathrm{a}$ & 4.62 & $1.30 \mathrm{a}$ & 0.05 \\
\hline & 10.17 & $57 \mathrm{a}$ & 8.25 & $21 \mathrm{a}$ & 8.25 & $3 a$ & 3.83 & $9 a$ & 3.83 & $1.44 \mathrm{a}$ & 0.11 \\
\hline \multirow{3}{*}{$\begin{array}{l}\text { Hedyosmum } \\
\text { luteynii }\end{array}$} & 5 & $37 a$ & 19.97 & $37 \mathrm{a}$ & 13.22 & $8 a$ & 4.62 & $7 a$ & 6.00 & $0.93 \mathrm{a}$ & 0.09 \\
\hline & 8 & $50 \mathrm{a}$ & 12.44 & $29 a$ & 10.52 & $4 a$ & 0.00 & $8 \mathrm{a}$ & 3.27 & $0.87 \mathrm{a}$ & 0.01 \\
\hline & 10.38 & $45 \mathrm{a}$ & 11.94 & $26 a$ & 7.66 & $6 a$ & 2.31 & $11 \mathrm{a}$ & 5.03 & $0.84 \mathrm{a}$ & 0.05 \\
\hline \multirow{3}{*}{$\begin{array}{c}\text { Oreopanax } \\
\text { avicenniifolius }\end{array}$} & 5 & $25 a$ & 5.03 & $4 a$ & 3.27 & $0 \mathbf{b}$ & 0.00 & $70 a$ & 6.93 & $2.29 a$ & 0.19 \\
\hline & 8 & $35 \mathrm{a}$ & 10.00 & $2 \mathrm{a}$ & 4.00 & $1 \mathbf{b}$ & 2.00 & $61 \mathrm{a}$ & 9.45 & $2.16 \mathrm{a}$ & 0.18 \\
\hline & 9.47 & $21 \mathrm{a}$ & 3.83 & $5 \mathrm{a}$ & 2.00 & $6 \mathbf{a}$ & 2.31 & $66 \mathrm{a}$ & 5.16 & $2.25 \mathrm{a}$ & 0.23 \\
\hline
\end{tabular}

Leyenda: $\mathrm{n}=4, \mathrm{DE}=$ desviación estándar, diferentes letras significan diferencias significativas entre grupos de acuerdo a Tukey $(\mathrm{p}<0.05)$.

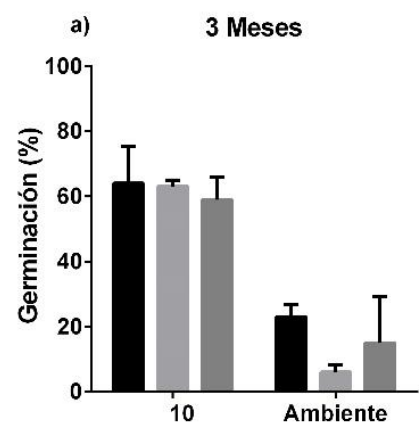

Temperatura $\left({ }^{\circ} \mathrm{C}\right)$
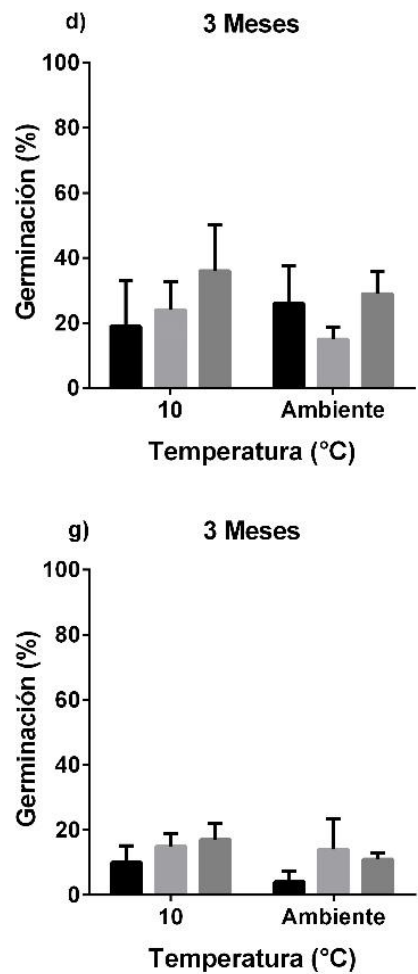

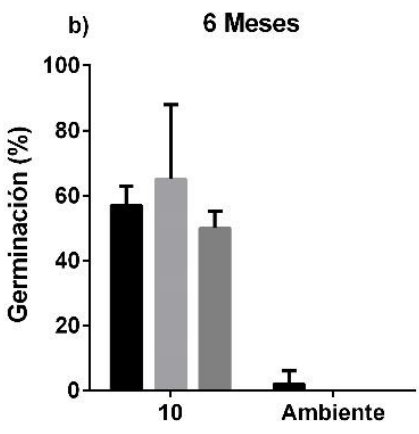

Temperatura $\left({ }^{\circ} \mathrm{C}\right)$
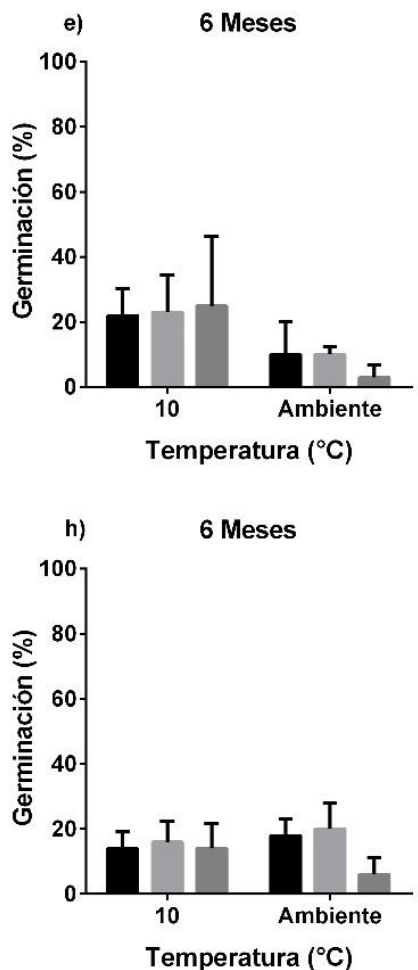

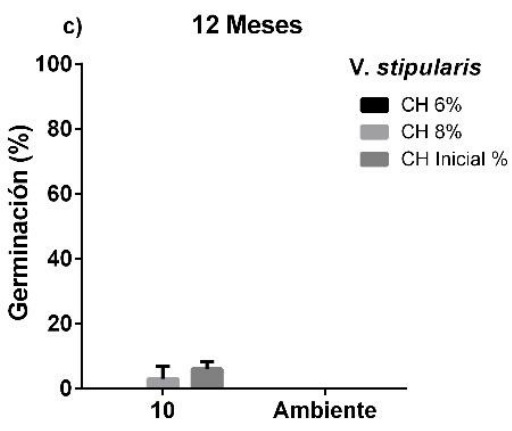

Temperatura $\left({ }^{\circ} \mathrm{C}\right)$
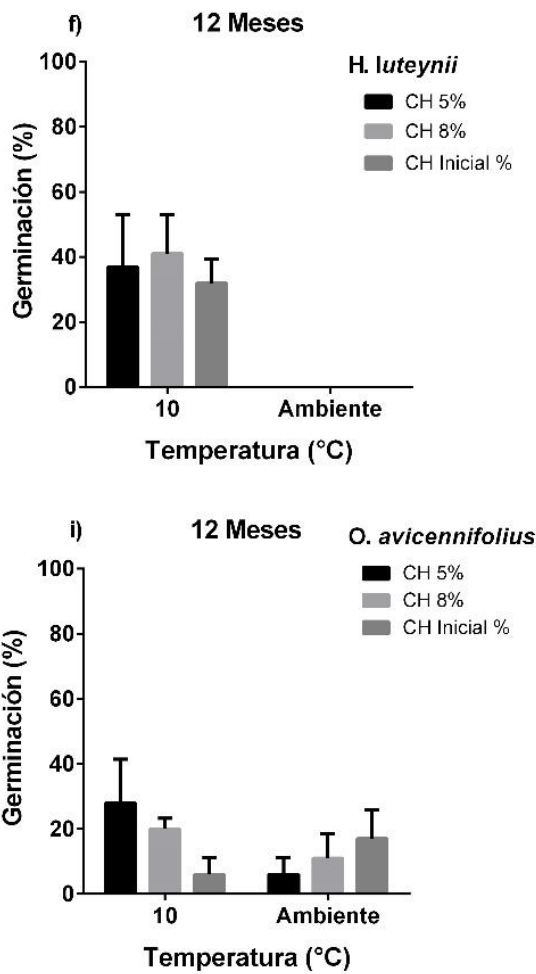

Figura 2. Porcentaje de germinación de Vallea stipularis (a,b,c), Hedyosmum luteynii (d,e,f) y Oreopanax avicenniifolius $(\mathrm{g}, \mathrm{h}, \mathrm{i})$, almacenadas en tres niveles de contenido de humedad $(\mathrm{CH})$ de las semillas, a $10^{\circ} \mathrm{C}$ y temperatura ambiente, durante 3,6 y 12 meses. Las barras representan el error estándar. 


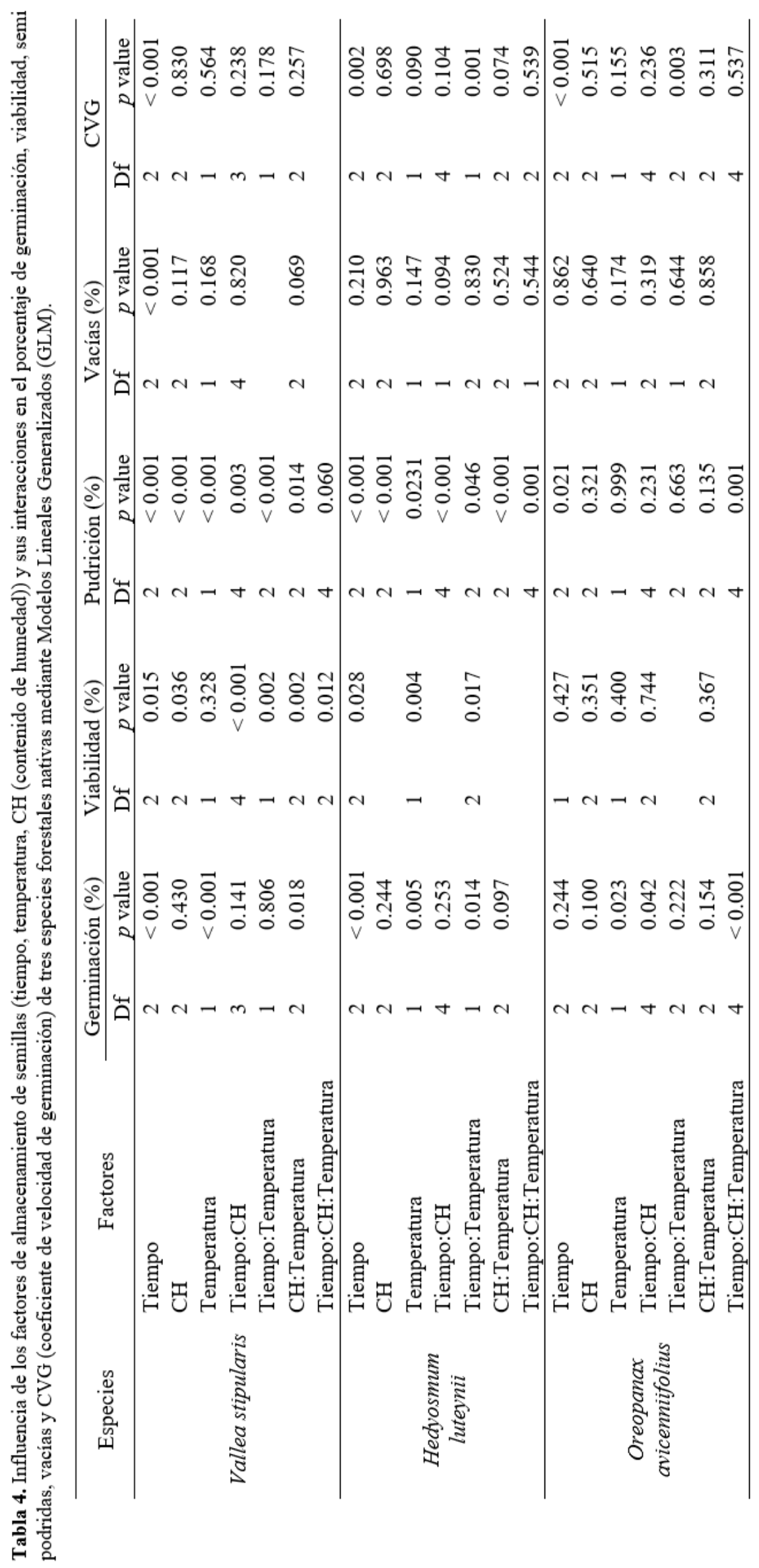



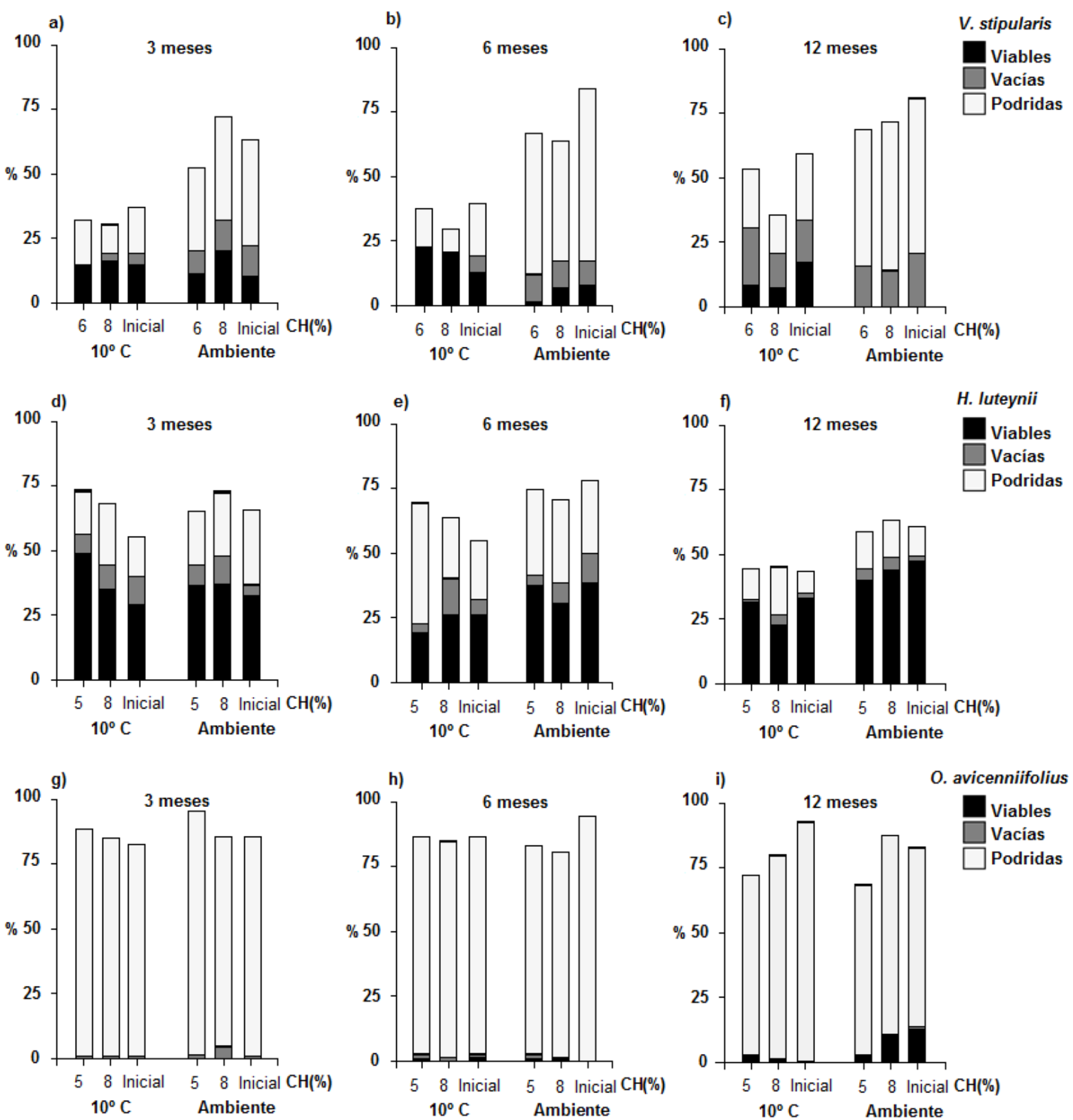

Figura 3. Porcentaje de semillas viables, vacías y podridas de Vallea stipularis (a,b,c), Hedyosmum luteynii (d,e,f) y Oreopanax avicenniifolius (g,h,i), almacenadas en tres niveles de contenido de humedad $(\mathrm{CH})$ de las semillas, a $10^{\circ} \mathrm{C} \mathrm{y}$ temperatura ambiente, durante 3,6 y 12 meses.

CH presentó valores bajos de germinación (2\%) (Fig. 2b), y tal como se esperaba, a los 12 meses la germinación fue nula (Fig. 2c). Los factores que estadísticamente influyeron en la germinación fue el tiempo y temperatura $(p<0.001)$ y la interacción entre $\mathrm{CH}$ y temperatura $(p=0.018)$ (Tabla 4).

En H. luteynii las semillas almacenadas durante 3,6 y 12 meses, a $10^{\circ} \mathrm{C}$ con diferentes niveles de contenido de humedad de las semillas, presentaron porcentajes de germinación que oscilaron entre 20 y $40 \%$, existiendo un patrón en el incremento del porcentaje de germinación a medida que transcurre el tiempo de almacenamiento (Fig. 2d,e,f). Por el contrario, los tratamientos de almacenamiento a temperatura ambiente, a partir del sexto mes de almacenamiento empezaron a disminuir su capacidad germinativa con valores menores al 10\% (Fig. 2e), y a los $12 \%$ de almacenamiento en los diferentes niveles de $\mathrm{CH}$, no se registró valores de germinación (Fig. 2f). Los factores que influenciaron significativamente fueron el tiempo $(p<0.001)$, la temperatura $(p=0.005)$, y su interacción ( $p=0.014)$ (Tabla 4).

En general, $O$. avicenniifolius presentó bajos porcentajes de germinación a los 3, 6 y 12 meses de almacenamiento (Fig. 2g,h,i), con valores máximos de germinación del $28 \%$ en semillas almacenadas a $10^{\circ} \mathrm{C}$ con $5 \%$ de $\mathrm{CH}$ a los 12 meses (Fig. 2i). El factor que influyó significativamente en la germinación fue la temperatura $(p=0.023)$ y las interacciones entre tiempo y $\mathrm{CH}$ $(p=0.042)$; y tiempo, $\mathrm{CH}$ y temperatura $(p<0.001)$ (Tabla $4)$. 


\subsection{Efecto de las diferentes condiciones de almacenamiento sobre el porcentaje de semillas viables, vacías y podridas de tres especies forestales nativas}

Las semillas de $V$. stipularis almacenadas durante 3 y 6 meses a $10^{\circ} \mathrm{C}$, con los tres niveles de $\mathrm{CH}$, presentaron porcentajes de viabilidad entre 15 a $20 \%$ (Fig. 3a,b), sin embargo, a los 12 meses existió un descenso de la viabilidad $(<10 \%)$ principalmente en semillas con contenidos de humedad del 6 y $8 \%$ (Fig. 3c). La viabilidad de las semillas almacenadas a temperatura ambiente fue descendiendo a medida que trascurría el tiempo de almacenamiento; de esta manera, a los 12 meses las semillas almacenadas bajo estas condiciones perdieron totalmente su viabilidad. En general, se observó que las semillas almacenadas a temperatura ambiente presentaron porcentajes más altos de pudrición con un valor máximo del 67\% (Fig. 3a,b,c). Por otro lado, se observó un bajo porcentaje de semillas vacías en las diferentes condiciones de almacenamiento (Fig. 3a,b,c), aunque tuvo un incremento principalmente a los 12 meses de almacenamiento (Fig. 3c). Los factores que influenciaron significativamente en la viabilidad fueron el tiempo $(p=0.015)$ y $\mathrm{CH}(p=0.036)$ y todas sus interacciones $(p<0.05)$ (Tabla 4). Para la variable pudrición tanto los factores solos y sus interacciones fueron estadísticamente significativos $(p<0.05)$, excepto la interacción de los tres factores $(p=0.060)$. Para las semillas vacías, el factor que mayor influenció fue el tiempo de almacenamiento $(p<0.001)$ (Tabla 4).

Las semillas de $H$. luteynii almacenadas durante 3 meses, a $10^{\circ} \mathrm{C}$, con los distintos niveles de $\mathrm{CH}$, presentaron porcentajes de viabilidad que oscilaron entre 29 a $48 \%$; a los 12 meses, existió un leve descenso que varió del 23 al $33 \%$ (Fig. 3d,f). Los tratamientos de almacenamiento a temperatura ambiente, pese a haber presentado niveles bajos o nulos de germinación, mantuvieron la viabilidad de las semillas entre 30 a 47\%, a los 3, 6 y 12 meses (Fig. $3 \mathrm{~d}, \mathrm{e}, \mathrm{f})$. Los factores que tuvieron influencia significativa en la viabilidad fue el tiempo $(p=0.028)$ y la temperatura $(p=0.004)$ y su interacción $(p=0.017)$. Para la variable pudrición se encontró diferencias significativas por cada factor evaluado y todas sus interacciones $(p<0.05) \mathrm{y}$ contrariamente para la variable semillas vacías no hubo significancia estadística $(p>0.05)$ (Tabla 4$)$.

Los resultados de viabilidad de $O$. avicenniifolius demostraron que debido a los altos porcentajes de pudrición superior al $65 \%$ en todos los tratamientos de almacenamiento, su viabilidad fue baja (Fig. 3g,h,i). No obstante, las semillas mantienen la viabilidad, aunque en bajos porcentajes (máximo 13\%) a los 12 meses de almacenamiento, especialmente a temperatura ambiente (Fig. 3i). No se encontró diferencias significativas para la variable viabilidad y semillas vacías $(p>0.05)$, mientras que para la variable pudrición, influyó significativamente el factor tiempo $(p=0.021)$ y la interacción entre los tres factores evaluados $(p=0.001)$ (Tabla 4$)$.

\subsection{Efecto de diferentes condiciones de almacenamiento sobre la velocidad de germinación de tres especies forestales nativas}

Las semillas de $V$. stipularis almacenadas, durante 3 y 6 meses, empezaron a germinar a partir de los 51 y 39 días después de la siembra respectivamente (Fig. 4a,b), con CVG que varió entre 1.4 a 1.6, mientras que a los 12 meses y $10^{\circ} \mathrm{C}$, los valores del CVG fueron los más bajos, lo que indica una germinación lenta y con bajos porcentajes de germinación (Tabla 5). Además, se observó que las semillas expuestas a $10^{\circ} \mathrm{C}$ con sus tres combinaciones de $\mathrm{CH}$ presentaron los mayores porcentajes de germinación acumulativa (superior al 50\%) principalmente a los tres meses (Fig. 4a). Lo contrario fue observado para las semillas almacenadas a temperatura ambiente, con todos los niveles de contenido de humedad, los cuales presentaron bajos porcentajes de germinación acumulativa en los periodos que se registró germinación, y tuvieron valores de CVG inferior al 1.1 a los 6 meses de almacenamiento (Fig. 4a,b) (Tabla 5). Se encontró que el tiempo de almacenamiento tuvo una significancia estadística para el CVG $(p<0.001)$ (Tabla 4).

En general, $H$. luteynii presentó una germinación tardía, cuyas semillas almacenadas a 3 y 6 meses, iniciaron su germinación a los 69 días después de la siembra y tuvieron valores de CVG de alrededor del 1.2 (Fig. 4d,e) (Tabla 5). También se encontraron valores inferiores a 0.9 principalmente a los 6 meses de almacenamiento (Tabla 5), lo que indica que en este periodo la germinación fue lenta, menor sincronía y bajos porcentajes de germinación acumulativa, especialmente en las semillas almacenadas a temperatura ambiente (Fig. 4e). A los 12 meses, las semillas almacenadas a $10^{\circ} \mathrm{C}$ y germinaron a los 73 días; sin embargo, presentaron los valores más altos de germinación acumulativa y los valores de CVG corresponden a 1.1 (Fig. 4f) (Tabla 5). Se encontró que el factor tiempo $(p=0.002)$ y su interacción con la temperatura $(p=0.001)$ tuvo una significancia estadística (Tabla 4).

Los resultados indicaron que las semillas de $O$. avicenniifolius iniciaron su germinación a partir de 47, 39 y 42 días después de la siembra para los periodos 3, 6 y 12 meses, respectivamente (Fig. 4g,h,i). En cuanto a los CVG, estos oscilaron entre 1.4 a 2.6, siendo los tratamientos de temperatura ambiente los que presentaron menores valores (Tabla 5), lo cual muestra que en esta temperatura las semillas tuvieron menor porcentaje de germinación acumulativa.

\section{DISCUSIÓN}

Con base a los resultados sobre el contenido de humedad inicial de las semillas y su respuesta al almacenamiento bajo diferentes condiciones, se demostró que las tres especies forestales nativas estudiadas corresponden a semillas ortodoxas (Jiménez \& Patiño, 2019), y además se notó que el comportamiento al almacenamiento depende de la especie, siendo la temperatura y el tiempo de almacenamiento, los factores más influyentes en las variables evaluadas.

$V$. stipularis, a los 12 meses de almacenamiento en condiciones de $10^{\circ} \mathrm{C}$; y a temperatura ambiente a partir del tercer mes de almacenamiento, se redujo drásticamente su capacidad germinativa, viabilidad y velocidad de germinación, e incluso al final del periodo de almacenamiento presentó valores nulos principalmente a temperatura ambiente. Estos hallazgos han sido reportados por otros autores, quienes indicaron que el almacenamiento afecta negativamente a la germinación, viabilidad y calidad de la semilla, debido al deterioro fisiológico natural de los embriones de las semillas, 
Tabla 5. Valores promedios de los coeficientes de velocidad de germinación (CVG) de tres especies forestales nativas almacenadas con tres niveles de contenido de humedad $(\mathrm{CH})$ de las semillas, a $10^{\circ} \mathrm{C}$ y temperatura ambiente, durante 3,6 y 12 meses.

\begin{tabular}{lcccccccccc}
\hline \multirow{2}{*}{ Tratamientos } & \multicolumn{3}{c}{ V. stipularis } & \multicolumn{3}{c}{ H. luteynii } & \multicolumn{3}{c}{ O. avicenniifolius } \\
\cline { 2 - 10 } & $3 \mathrm{~m}$ & $6 \mathrm{~m}$ & $12 \mathrm{~m}$ & $3 \mathrm{~m}$ & $6 \mathrm{~m}$ & $12 \mathrm{~m}$ & $3 \mathrm{~m}$ & $6 \mathrm{~m}$ & $12 \mathrm{~m}$ \\
\hline T. Ambiente_CH Inicial & 1.5 & SG & SG & 1.2 & 0.5 & SG & 2.2 & 1.9 & 2.0 \\
T. Ambiente_8\% CH & 1.6 & SG & SG & 1.2 & 1.1 & SG & 2.2 & 2.6 & 1.4 \\
T. Ambiente_6 o 5\% CH & 1.5 & 0.3 & SG & 1.2 & 0.8 & SG & 1.7 & 2.4 & 1.4 \\
$10^{\circ} \mathrm{C}$ CH Inicial & 1.5 & 1.5 & 0.9 & 1.2 & 0.9 & 1.1 & 2.2 & 2.4 & 1.5 \\
$10^{\circ} \mathrm{C}$ C8\% CH & 1.4 & 1.4 & 1.1 & 1.1 & 1.2 & 1.1 & 2.1 & 2.6 & 2.2 \\
$10^{\circ} \mathrm{C}$ 6 o 5\% CH & 1.5 & 1.5 & SG & 0.8 & 1.2 & 1.1 & 2.2 & 2.4 & 2.3 \\
\hline
\end{tabular}

$\mathrm{SG}=$ Sin germinación

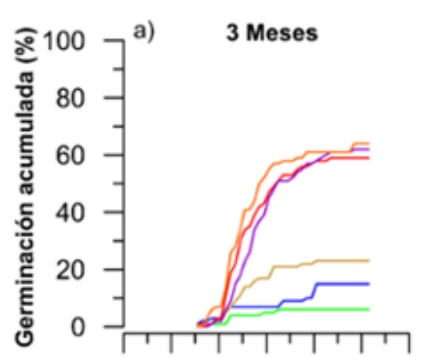

20406080100120140 Dias

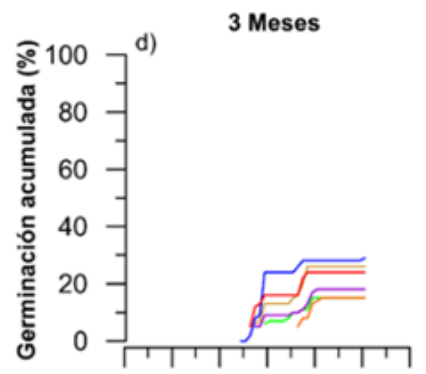

20406080100120140 Dias

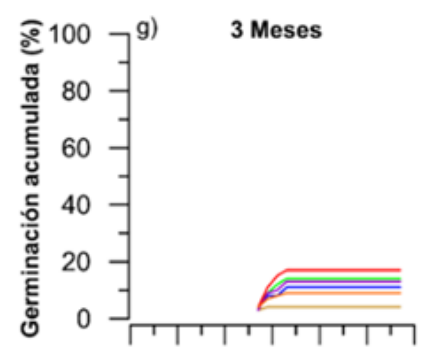

$20 \quad 3040 \quad 5060 \quad 7080$

Dias

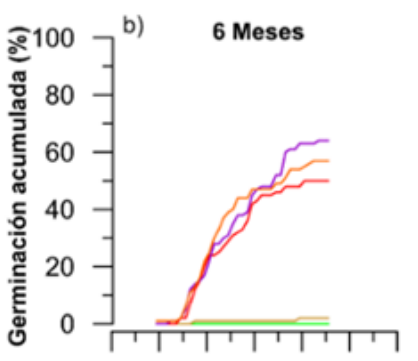

$2040 \quad 60 \quad 80100120140$

Días

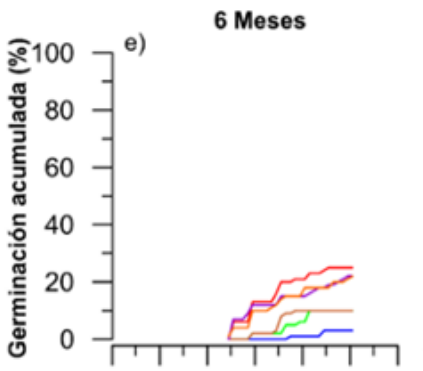

$20 \quad 4060 \quad 80100120140$

Dias

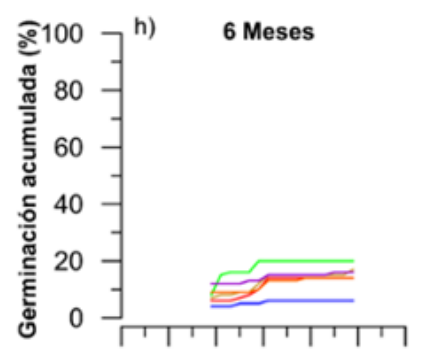

$\begin{array}{lllllll}20 & 30 & 40 & 50 & 60 & 70 & 80\end{array}$

Días

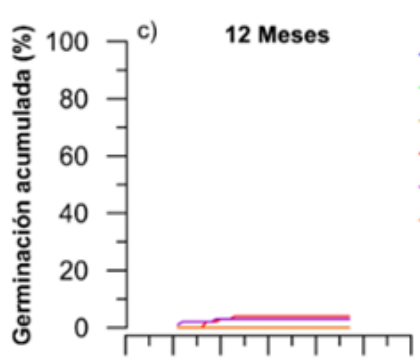

20406080100120140

Dias

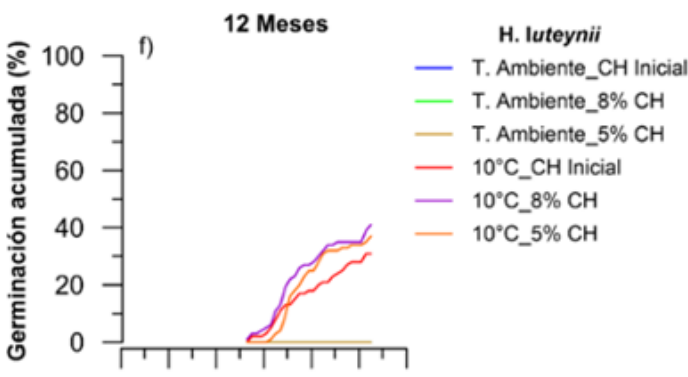

20406080100120140

Dias

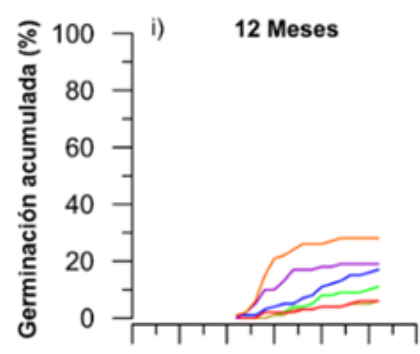

O. avicennifolius

- T. Ambiente_CH Inicial

- T. Ambiente_ $8 \% \mathrm{CH}$

- T. Ambiente_5\% $\mathrm{CH}$

- $10^{\circ} \mathrm{C} \_\mathrm{CH}$ Inicial

$-10^{\circ} \mathrm{C} \_8 \% \mathrm{CH}$

$-10^{\circ} \mathrm{C}-5 \% \mathrm{CH}$

Figura 4. Porcentaje de germinación acumulativa de Vallea stipularis (a,b,c), Hedyosmum luteynii (d,e,f) y Oreopanax avicenniifolius $(\mathrm{g}, \mathrm{h}, \mathrm{i})$, almacenadas con tres niveles de contenido de humedad $(\mathrm{CH})$ de las semillas, a $10^{\circ} \mathrm{C}$ y temperatura ambiente, durante 3,6 y 12 meses.

principalmente como resultado de los cambios de proteínas, la formación de radicales libres y oxidación de lípidos en las semillas durante el almacenamiento (Bailly, 2004; Pukacka \& Ratajczak, 2007; Çakmak et al., 2010; Shahidi \& Zhong, 2010; Rajjou et al., 2012; Bourgeois, Lemay, Landry, Rochefort, \& Poulin , 2019; Tian, Lv, Yuan, Zhang, \& Hu, 2019).
H. luteynii a $10^{\circ} \mathrm{C}$, durante 12 meses de almacenamiento, mantuvo porcentajes de germinación y viabilidad similares a los valores iniciales de semillas sin almacenamiento. Además, se evidenció un incremento de la germinación a medida que transcurre el tiempo de almacenamiento; esto sugiere que las semillas pueden tener dormancia (Varela \& Arana, 2011). Courtis (2013) menciona que algunas especies presentan embriones 
morfológicamente completos, pero fisiológicamente inmaduros lo que repercute en la capacidad germinativa. Conforme el embrión alcanza un estado de madurez fisiológica, la dormancia disminuye (Ceballos \& López, 2007; Jimenez, Alche, Wang, \& Rodríguez, 2007). Shu, Liu, Xie, \& He (2016) indican que, durante la maduración, el embrión se mantiene en un estado de reposo, no moviliza casi ningún nutriente almacenado y no presenta división celular, ni alargamiento. Además, Née, Xiang, \& Soppe (2017) manifiestan, que las semillas recién cosechadas tienen una latencia relativamente alta y se libera gradualmente durante el almacenamiento de semillas. Por otra parte, nuestros resultados indican que la alta germinación de semillas almacenadas a bajas temperaturas $\left(10^{\circ} \mathrm{C}\right)$ en el último periodo evaluado, se debe a que probablemente hay una disminución del Ácido Abscísico (ABA) endógeno presente en las semillas con latencia (Baskin \& Baskin, 2001, 2003).

Los tratamientos de almacenamiento a temperatura ambiente de $H$. luteynii, a partir del sexto mes, empezaron a disminuir su capacidad germinativa y a los 12 de almacenamiento, no se registró valores de germinación. Por lo tanto, el almacenamiento a condiciones de temperatura ambiental no beneficia a $V$. stipularis y $H$. luteynii. De ahí que, Ceballos \& López (2007), señalan que las semillas almacenadas a esta temperatura continúan con los procesos de respiración e incrementan la tasa metabólica, dando lugar a la oxidación o destrucción de algunos lípidos, proteínas y enzimas, causando el deterioro de la semilla y hasta la muerte del embrión, afectando la germinación. Los resultados obtenidos coinciden con un estudio realizado por Procházková \& Bezděčková (2008) en Fagus sylvatica, quienes concluyen que almacenar estas semillas a $20^{\circ} \mathrm{C}$ tiene un efecto negativo en su germinación.

Por otro lado, a pesar de que $H$. luteynii tuvo un porcentaje de germinación reducido en condiciones de almacenamiento a temperatura ambiente, la viabilidad de sus semillas almacenadas fue alta hasta los 12 meses de almacenamiento, esto sugiere que la dormancia puede ser en un tiempo superior al año, lo cual condiciona la germinación. Uno de los factores que explican este comportamiento, es que hay un aumento endógeno de ABA y una menor concentración de giberelinas (GA) en las semillas (Kudred \& Sener, 1990; Ali et al., 2004; Kucera, Cohn, \& Leubner-Metzger, 2005). Conforme a un estudio realizado por Ribeiro \& Costa (2015), recomiendan someter a las semillas a condiciones de baja temperatura para estimular la germinación, pues se conoce que la estratificación fría es un método recomendado para romper la dormancia, principalmente especies adaptadas a climas fríos. Por tanto, más investigaciones son necesarias para acelerar la germinación, pues se conoce que, en función de la temperatura de almacenamiento, la dormancia puede aumentar o desaparecer (Geneve, 2003).

Las semillas de $O$. avicenniifolius, presentaron geminación más temprana, con mayor sincronización, pero con bajos porcentajes de germinación y altos de pudrición. Siddique \& Wright (2003), afirman que cuando el contenido de humedad es demasiado alto, las semillas pueden ser susceptible a la contaminación por hongos, pero esto no coincide con los resultados del contenido de humedad para esta especie, debido a que su valor inicial está por debajo del 10\%. De ahí que, Schmidt (2000) manifiesta que la baja calidad genética de las semillas, puede ser un factor determinante en el deterioro y aumento de la susceptibilidad por la infección de hongos. Por tanto, varios autores sugieren que las colecciones de semillas deben considerar la variabilidad genética en una población y su calidad de semillas, para evitar problemas en el almacenamiento y por ende en la germinación (Lengkeek, Jaenicke, \& Dawson, 2005; Pakkad et al., 2008; Thomas et al., 2014).

\section{CONCLUSIONES}

En general, los factores independientes que jugaron un rol importante en la conservación de las semillas fueron el tiempo y la temperatura, mientras que la reducción del contenido de humedad de semillas no influyó en el mantenimiento de la viabilidad de las semillas. A nivel de especies, hubo una respuesta diferente al almacenamiento, aunque $V$. stipularis y $H$. luteynii, claramente pierden su capacidad de germinación en condiciones de almacenamiento a temperatura ambiente y conforme pasa el tiempo de almacenamiento. O. avicenniifolius presentó una germinación temprana y rápida, pero con bajos porcentajes de germinación debido a su alta pudrición. Sin embargo, mantuvo su capacidad germinativa hasta los 12 meses de almacenamiento en las dos temperaturas evaluadas. A pesar de los hallazgos de este estudio, otras investigaciones son necesarias en el contexto del almacenamiento de semillas, pues se debe considerar la evaluación de la respuesta de las semillas en tiempos más prolongados, a menor temperatura de almacenamiento e incluir más especies forestales con fines de conservación y restauración en experimentos de almacenamiento. Así como también evaluar la calidad de plántula posterior al almacenamiento es necesario.

\section{AGRADECIMIENTOS}

Este trabajo presentando estuvo en el marco del proyecto de investigación "Efectos del cambio climático en la capacidad germinativa de semillas y producción de plántulas de especies forestales nativas en la provincia del Azuay", ganador del XIII Concurso de Proyectos de investigación de la Dirección de Investigación de la Universidad de Cuenca. Los autores agradecen al Ministerio del Ambiente (Zonal 6) y a la Empresa Pública Municipal de Telecomunicaciones, Agua Potable, Alcantarillado y Saneamiento de Cuenca (ETAPA), por los permisos de investigación y la coordinación mensual para el ingreso a los bosques de Mazán y Llaviucu, respectivamente. Un agradecimiento para Andrea Maza y Shannon Serpa por su apoyo en el laboratorio.

\section{REFERENCIAS}

Ali, S., Bouinot, D., Wagner, M. H., Bonnet, M., Sotta, B., Grappin, P., \& Jullien, M. (2004). Changes in endogenous abscisic acid levels during dormancy release and maintenance of mature seeds: Studies with the Cape Verde Islands ecotype, the dormant model of Arabidopsis thaliana. Planta, 219(3), 479-488. https://doi.org/10.1007/s00425-004-1251-4 
Bailly, C. (2004). Active oxygen species and antioxidants in seed biology. Seed Science Research, 14(2), 93-107. https://doi.org/10.1079/ssr2004159

Baskin, C. C., \& Baskin, J. M. (2001). Seeds: ecology, biogeography, and evolution of dormancy and germination. San Diego, USA: Academic Press.

Baskin, C. C., \& Baskin, J. M. (2003). Seed germination and propagation of Xyris tennesseensis a federal endamgered wetland species. Wetlands (Vol. 23). doi:https://doi.org/10.1672/02775212(2003)023[0116:sgapox]2.0.co;2

Baskin, C. C., \& Baskin, J. M. (2014). Variation in seed dormancy and germination within and between individuals and populations of a species. Seeds: Ecology, biogeography and evolution of dormancy and germination. https://doi.org/10.1016/B978-0-124166776.00008-1

Bates, D., Machler, M., Bolker, B., \& Walker, S. (2015). Fitti VCFR4ZAng linear mixed-effects models using lme4. Journal of Statistical Software, 67, 1-48. http://dx.doi.org/10.18637/ jss.v067.i01

Bewley, J. D., Bradford, K. J., Hilhorst, H. W. M., \& Nonogaki, H. (2013). Seeds: physiology of development, germination and dormancy. New York, USA: Springer.

Bourgeois, B., Lemay, M. A., Landry, T., Rochefort, L., \& Poulin, M. (2019). Seed storage behaviour of eight peatland pool specialists: Implications for restoration. Aquatic Botany, 152, 59-63. https://doi.org/10.1016/j.aquabot.2018.09.008

Çakmak, T., Atici, O., Agar, G., \& Sunar, S. (2010). Natural aging-related biochemical changes in alfalfa (Medicago sativa L.) seeds stored for 42 years. International Research Journal of Plant Science, 1(1), 001-006.

Ceballos, Á., \& López, J. (2007). Conservación de la calidad de semillas forestales nativas. Almacenamiento. 58(4), 265-292.

Courtis, A. (2013). Germinación de semillas: Cátedra de fisiología vegetal carreras. Universidad Nacional del Nordeste, Argentina, 1-22. Disponible en https://docplayer.es/12165022-Catedra-de-fisiologiavegetal.html

Defries, R. S., Rudel, T., Uriarte, M., \& Hansen, M. (2010). Deforestation driven by urban population growth and agricultural trade in the twenty-first century. Nature Geoscience, 3(3), 178-181. https://doi.org/10.1038/ngeo756

Doria, J. (2010). Generalidades sobre las semillas: su producción, conservación y almacenamiento. AGRIS, 31(1), 74-85.

Estrella, J., Manosalvas, R., Mariaca, J., \& Ribadeneira, M. (2005). Biodiversidad y recursos genéticos: Una guía para su uso y acceso en el Ecuador. Quito, Ecuador: EcoCiencia.

FAO. (2012). El estado de los recursos geneticos forestales en el mundo. Informe Nacional Ecuador. Quito. Disponible en http://www.fao.org/3/i3825e/i3825e20.pdf

FAO, FIDA, \& PMA. (2014). El estado de la inseguridad alimentaria en el mundo 2014. Fortalecimiento de un entorno favorable para la seguridad alimentaria y la nutrición. Rome, Italy: FAO. Disponible en http://www.fao.org/3/a-i4030s.pdf

Gentil, D. F. de O. (2001). Conservao de sementes do cafeeiro: Resultados discordantes ou complementares? Bragantia, 60(3), 149-154.

Geneve, R. L. (2003). Impact of temperature on seed dormancy. HortScience: a publication of the American Society for Horticultural Science, 38(3), 336-341. doi:10.21273/HORTSCI.38.3.336

Grijalva, J., Ximena, C., Ramos, R., Barrera, P., Vera, R., \& Sigcha, F. (2015). Estado de los recursos genéticos forestales en Ecuador. Programa Nacional de Forestería del Instituto Nacional de Investigaciones Agropecuarias. (Publicació). Retrieved from http://181.112.143.123/bitstream/41000/2827/1/iniapsc 322est.pdf

ISTA. (2007). International rules for seed testing (Vol. 5). https://www.seedtest.org/en/home.html

Jimenez, J., Alche, J., Wang, W., \& Rodríguez, M. (2007). Alpeorujo y semillas de olivo presentan el mismo tipo de proteinas de almacenamiento. En: Olivar, F. D. (Ed.), Simposium Científico Técnico del Año 2005, (págs. 365 - 376). doi:978-84-934503-0-4

Jiménez, J., \& Patiño, C. (2019). Germinación, desarrollo inicial y supervivencia de plántulas bajo diferentes condiciones de almacenamiento de semillas de tres especies nativas de bosques del Parque Nacional Cajas (Universadad de Cuenca). Retrieved from http://dspace.ucuenca.edu.ec/handle/123456789/32802

Kindt, R., Lilles $\varnothing$, J.-P. B., Mbora, A., Muriuki, J., Wambugu, C., Frost, W., ... Holding-Anyonge, C. (2006). Tree seeds for farmers: a toolkit and reference source. Nairobi, Kenya. Disponible en http://www.worldagroforestry.org/output/tree-seedsfarmers-toolkit-and-reference-source

Kucera, B., Cohn, M. A., \& Leubner-Metzger, G. (2005). Plant hormone interactions during seed dormancy release and germination. Seed Science Research, 15(4), 281-307. https://doi.org/10.1079/SSR2005218

Kudred, K., \& Sener, B. (1990). Effects of kinetin and gibberellic acid in overcoming high temperature and salinity $(\mathrm{NaCl})$ stresses on the germination of barley and lettuce. Seeds Phyton (Horn, Austria), 30(1), 6574.

Lengkeek, G., Jaenicke, H., \& Dawson, K. (2005). Genetic bottlenecks in agroforestry systems: Results of tree nursery surveys in East Africa. Agroforestry Systems, 63(2), 149-155. https://doi.org/10.1007/s10457-004-9155-7

Ministerio del Ambiente. (2019). Plan Nacional de Restauración Forestal 2019-2030. Quito, Ecuador.

Myers, N., Mittermeier, R. A., Mittermeier, C. G., da Fonseca, G. A. B., \& Kent, J. (2000). Biodiversity hotspots for conservation priorities. Nature, 403(6772), 853-858. https://doi.org/10.1038/35002501

Née, G., Xiang, Y., \& Soppe, W. J. (2017). The release of dormancy, a wake-up call for seeds to germinate. Current Opinion in Plant Biology, 35, 8-14. https://doi.org/10.1016/j.pbi.2016.09.002

Palomeque, X., Maza, A., Iñamagua, J. P., Günter, S., Hildebrandte, P., Weber, M., Stimm, B. (2017). Variabilidad intraespecífica en la calidad de semillas de especies forestales nativas en bosques montanos en 
el sur del Ecuador: Implicaciones para la restauración de bosques. Revista de Ciencias Ambientales (Trop J Environ Sci), 51(2), 52-72.

Pakkad, G., Al Mazrooei, S., Blakesley, D., James, C., Elliott, S., Luoma-Aho, T., \& Koskela, J. (2008). Genetic variation and gene flow among Prunus cerasoides D. Don populations in northern Thailand: Analysis of a rehabilitated site and adjacent intact forest. New Forests, 35(1), 33-43. https://doi.org/10.1007/s11056-007-9059-2

Pammenter, N. W., \& Berjak, P. (2000). Semillas Ortodoxas y Recalcitrante. Obtenido de Manual de Semillas de Árboles Tropicales: https://rngr.net/publications/manual-de-semillas-dearboles-tropicales/parte-i/semillasortodoxas_yrecalcitrantes/at_download/file

Pinheiro, J., Bates, D., DebRoy, S., \& Sarkar, D. (2018). nlme: Linear and nonlinear mixed effects models. $R$ package version 3.5.1. Obtenido de https://CRAN.Rproject.org/package $=$ nlme.

Pritchard, H. W., \& Dickie, J. B. (2003). Predicting seed longevity: the use and abuse of seed viability equations. Chapter 35, 1-70. Disponible en https://www.academia.edu/17275313/ Predicting_seed_longevity_the_use_and_abuse_of_see d_viability_equations?auto=download

Pritchard, H. W. (2004). Classification of seed storage types for ex situ conservation in relation to temperature and moisture. Washington D.C., USA.

Procházková, Z., \& Bezděčková, L. (2008). Effects of moisture content, storage temperature and type of storage bag on the germination and viability of stored European beech (Fagus sylvatica L.) seeds. Journal of Forest Science, 54(7), 287-293.

Pukacka, S., \& Ratajczak, E. (2007). Age-related biochemical changes during storage of beech (Fagus sylvatica L.) seeds. Seed Science Research, 17(1), 4553. https://doi.org/10.1017/S0960258507629432

Rajjou, L., Duval, M., Gallardo, K., Catusse, J., Bally, J., Job, C., \& Job, D. (2012). Seed Germination and Vigor. Annual Review of Plant Biology, 63(1), 507533. https://doi.org/10.1146/annurev-arplant-042811105550

R Core Team. (2018). R: A language and environment for statistical computing. Disponible en https://www.Rproject.org/

Ribeiro, J., \& Costa, C. (2015). The effect of temperature regulation on seed germination of the tropical tree Myrsine parvifolia A. DC near its southern limit. South African Journal of Botany, 98, 128-133. https://doi.org/10.1016/j.sajb.2015.02.012

Romero-Saritama, J. M. (2018). Seed conservation: An alternative to store germplasm and recover threatened Ecuadorian forests. Neotropical Biology and Conservation, 13(1), 74-85. https://doi.org/10.4013/nbc.2018.131.09
Royal Botanic Gardens Kew. (2015). Seed Information Database(SID). Version 7.1. Disponible en http://data.kew.org/sid/

Schmidt, L. H. (2000). Guide to handling of tropical and subtropical forest seed. Danida Forest Seed Centre, Hoersholm, Denmark.

Shahidi, F., \& Zhong, Y. (2010) Lipid oxidation and improving the oxidative stability. Chemical Society Reviews, 39, 4067-4079. http://dx.doi.org/10.1039/b922183m

Shu, K., Liu, X. D., Xie, Q., \& He, Z. H. (2016). Two faces of one seed: Hormonal regulation of dormancy and germination. Molecular Plant, 9(1), 34-45. https://doi.org/10.1016/j.molp.2015.08.010

Siddique, A. B., \& Wright, D. (2003). Effects of different seed drying methods on moisture percentage and seed quality (viability and vigour) of Pea Seeds (Pisum sativum L.). Asian Journal of Plant Sciences, 2(13), 978982. https://doi.org/10.3923/ja.2003.201.208

Sierra, R. (1999). Propuesta preliminar de un sistema de clasificación de vegetación para el Ecuador Continental [Preliminary proposal of a vegetation classification system for Continental Ecuador]. 175 p. https://doi.org/10.13140/2.1.4520.9287

Smith, J. R., Mengistu, A., Nelson, R. L., \& Paris, R. L. (2008). Identification of soybean accessions with high germinability in high-temperature environments. Crop Science, 48(6), 2279-2288. https://doi.org/10.2135/cropsci2008.01.0026

Thomas, E., Jalonen, R., Loo, J., Boshier, D., Gallo, L., Cavers, S., ... Bozzano, M. (2014). Forest ecology and management genetic considerations in ecosystem restoration using native tree species. Forest Ecology and Management, 333(2014), 66-75. https://doi.org/10.1016/j.foreco.2014.07.015

Tian, P. P., Lv, Y. Y., Yuan, W. J., Zhang, S. B., \& Hu, Y. Sen. (2019). Effect of artificial aging on wheat quality deterioration during storage. Journal of Stored Products Research, 80, 50-56. https://doi.org/10.1016/j.jspr.2018.11.009

Varela, S., \& Arana, V. (2011). Latencia y germinación de semillas. Tratamientos pregerminativos. Serie Técnica - Sistemas Forestales Integrados, 3(1), 10.

Velasquez, J. S., Montero, A. R., \& Tapia, C. G. (2008). Semillas tecnología de producción y conservación. Quito, Ecuador: INIAP, Estación Experimental Santa Catalina, Departamento de Producción de Semillas. Disponible en http://repositorio.iniap.gob.ec/bitstream/41000/ 56/1/iniapsc280.pdf

Walters, C., Wheeler, L. M., \& Grotenhuis, J. (2005). Longevity of seeds stored in a gene bank: species characteristics. Seed Science Research, 15(1), 1-20. https://doi.org/10.1079/SSR2004195 